[Original Article]

\title{
EFFECT OF METHOTREXATE ON FRACTURE HEALING
}

\author{
KOICHIRO SATOH ${ }^{1)}$, HANS MARK $^{2}$, PETER ZACHRISSON ${ }^{2}$, \\ BJÖRN RYDEVIK ${ }^{3)}$, GUNNAR BYRÖD ${ }^{2)}$, SHIN-ICHI KIKUCHI ${ }^{1)}$, \\ SHIN-ICHI KONNO ${ }^{1)}$ and MIHO SEKIGUCHI ${ }^{1)}$ \\ ${ }^{1)}$ Department of Orthopaedic Surgery, Fukushima Medical University School of Medicine, Fukushima, \\ Japan, ${ }^{2)}$ Department of Plastic Surgery, Sahlgrenska University Hospital, Gothenburg, Sweden, ${ }^{3)}$ Depart- \\ ment of Orthopaedic Surgery, Göteborg University, Gothenburg, Sweden
}

(Received July 22, 2010, accepted December 27, 2010)

\begin{abstract}
Low doses of methotrexate (MTX) are safe and effective for treating adult and juvenile rheumatoid arthritis. However, because this powerful anti-inflammatory drug might negatively influence the healing of wounds and fractures, MTX administration is often stopped during surgical procedures. The present study assesses the effects of low- and high-dose MTX on early inflammatory processes and bone healing in an experimental model of fracture.

Thirty male Sprague-Dawley rats were assigned to low- and high-dose MTX and control groups. A femur was cut using a reciprocating saw and a 2-mm fracture gap was made using a fixator. One or four weeks thereafter, macrophages were immunostained and new bone formation was histomorphometrically measured.

Significantly less new bone was formed in the high-dose MTX, than in the control group $(p<0.01)$, whereas bone formation did not significantly differ between the low-dose MTX and control groups. These results suggested that a low dose of MTX does not affect the early process of endochondral bone formation during fracture healing, whereas a high dose might delay the progress of new periosteal bone formation. Although more macrophages were found in the groups treated with MTX, their impact on surrounding inflammatory processes remains unclear.
\end{abstract}

Key words : Fracture healing, Methotrexate, bone formation

\section{INTRODUCTION}

Low-dose methotrexate (MTX ; 10-25 mg/week) is considered the first choice of disease-modifying anti-rheumatic drugs (DMARD) for treating adult and juvenile rheumatoid arthritis, as well as other inflammatory systemic diseases. Low weekly doses of MTX have proven to be a safe and effective strategy with relatively few side effects. Methotrexate was originally developed to treat malignancies and it works by competitively inhibiting dehydrofolate reductase and blocking de novo purine synthesis, which interferes with folate metabolism in proliferating malignant cells. However, the pharmacological mec- hanisms underlying the powerful anti-inflammatory properties of MTX are not well understood. Serum levels of inflammatory cytokines are diminished and MTX also affects T-cell function. Increased levels of the anti-inflammatory nucleoside adenosine might be responsible for the effects of MTX on inflammatory disorders ${ }^{1)}$.

The risk of osteoporosis, bone pain and spontaneous fractures increases in patients with cancers who repeatedly receive high doses of MTX (a total dose of MTX>500 mg) ${ }^{2,3)}$. Because of these findings, and also as a response to clinical rheumatological studies indicating an increased risk of postoperative wound infections ${ }^{4-6)}$, ongoing anti-rheumatic

佐藤弘一郎, Hans Mark, Peter Zachrisson, Björn Rydevik, Gunnar Byröd, 菊地臣一, 紺野慎一, 関口美穂

Corresponding author : Miho Sekiguchi M.D., PhD E-mail address : miho-s@fmu.ac.jp

http://www.jstage.jst.go.jp/browse/fms http://fmu.ac.jp/home/lib/F-igaku/ 
therapy with MTX is often withheld from arthritic patients during surgery. This can cause disease activity to flare up, leading to increased pain, joint stiffness and problems during rehabilitation.

Bone healing after a fracture or surgery is initiated by a local inflammatory reaction. The extent to which such inflammatory processes and the subsequent formation of new bone are affected by potent anti-inflammatory drugs, such as MTX, in patients with arthritis and in healthy individuals remains unknown.

The present study assesses the early process of fracture healing under low- and high-dose MTX administration to evaluate the temporal distribution of endochondral bone formation during fracture healing in an experimental fracture model using healthy rats. The follow-up durations were selected to evaluate the effects of MTX upon the early inflammatory phase of fracture healing (1 week), and upon the decline of that phase with the formation of a high magnitude of endochondral bone ( 4 weeks) ${ }^{7,8}$.

The evaluation included histomorphometric analysis of de novo bone formation and an assessment of local inflammation in muscle tissues near the fracture area by immunostaining inflammatory cells (tissue macrophages).

\section{MATERIALS AND METHODS}

\section{Animals and anaesthesia}

Thirty male Sprague-Dawley rats weighing 350 $\mathrm{g}$ were assigned to the following groups.

Low-dose MTX (LDM1 and LDM4; $n=6$ each) : intraperitoneal injection of $3 \mathrm{mg} / \mathrm{kg} /$ week of MTX for 4 weeks before surgery and postoperative follow-up for 1 and 4 weeks, respectively. The MTX doses were selected based on previous experimental findings ${ }^{9-11)}$. Control (Con1 and Con $4 ; n=6$ each): intraperitoneal sham injection of saline (1 $\mathrm{mL} /$ week) for 4 weeks before surgery and follow up for 1 and 4 weeks thereafter, respectively. Low ${ }^{-}$ dose MTX was administered once per week in this study according to the treatment schedule for rheumatoid arthritis. High-dose MTX (HDM ; $n=6$ ) positive control : a bolus intraperitoneal injection of $250 \mathrm{mg} / \mathrm{kg}$ of MTX on the day of surgery. Highdose MTX is used to treat malignancies such as leukaemia and because the animals might not be able to tolerate such a regimen for over four weeks, a bolus was administered in the present study.

The animals were anaesthetised with an intraperitoneal injection of $1 \mathrm{~mL}$ of $\operatorname{Ketalar}^{\circledR}$ (50 mg/
$\mathrm{mL}$; Park Davis AB, Solna, Sweden) and Stesolid ${ }^{\circledR}$, (5 mg/mL, Dumex Alpharma AB, Stockholm, Sweden) in a $4: 1$ ratio. Analgesia was maintained via a subcutaneous injection of $0.01-0.05 \mathrm{mg} / \mathrm{kg}$ body weight of Temgesic ${ }^{\circledR}$, (Meda Sverige AB, Göteborg, Sweden) administered after surgery. The animals were housed in pairs after surgery without restrictions on weight-bearing or activities. Water and food was provided ad libitum and the rats were observed daily.

The research ethics committee at our institution approved the study protocols.

\section{Experimental fracture model}

Surgery proceeded according to the model developed by Mark et al. for experimental, external fixation of fractures in rat long bones ${ }^{7,8)}$. A curved incision was cut through the skin from the base of the tail to the knee to create a skin flap. The quadriceps and the hamstrings were gently dissected. A self-locking nylon strap was pulled through a tunnel dissected in the muscle around the mid portion of the femur to temporarily fix a drill guide on the lateral aspect of the bone. Four $0.8^{-}$ $\mathrm{mm}$ holes were drilled during irrigation and tapped. Four $1.2-\mathrm{mm}$ pins were inserted through the holes in the bone and cannulated through the skin flap. A fixator was fastened to the pins at a preset distance from the bone surface offset. An osteotomy was positioned between the two middle pins using a reciprocating saw and irrigation. The bone fragments were moved to a 2 -mm fracture gap using a screw for distraction or compression of the fixator (Fig. 1).

\section{Histological preparation}

We sacrificed the LDM1, Con1 and HMD groups after 1 week and the LDM4 and Con 4 groups after 4 weeks and perfusion-fixed each rat using PLP solution. The fractured limbs were dissected free of intact surrounding soft tissue and the fixator was removed from an intact pin-bone interface. Femoral specimens were immersion-fixed overnight in PLP solution at $4^{\circ} \mathrm{C}$ and then decalcified for 4 weeks in 15\% EDTA in PBS buffer that was changed twice each week. The pins were removed after decalcification, and then the specimens were cut in half transversely at the level of the proximal and distal pinholes and centrally in the sagittal plane. The specimens were dehydrated, embedded in paraffin, cut in 5- $\mu \mathrm{m}$-thick sections and further processed for histomorphometry or immunostaining. 


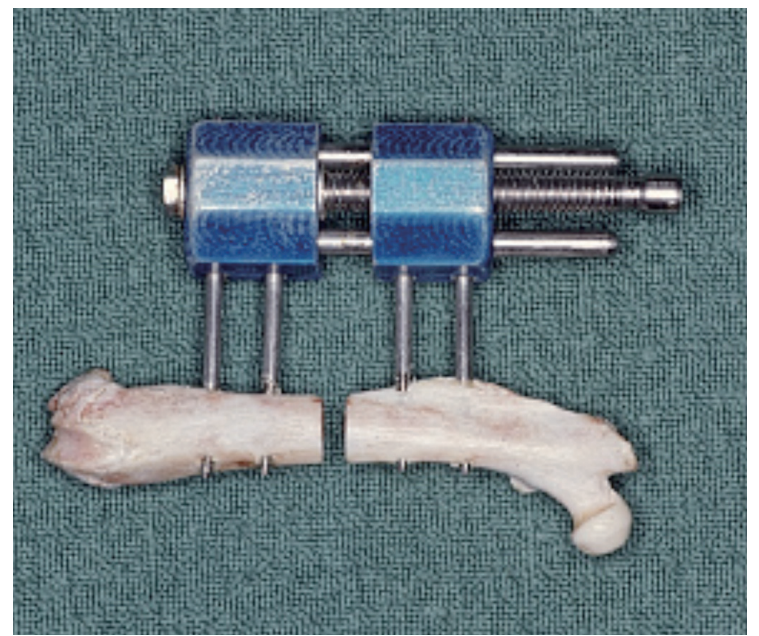

Fig. 1. Example of external fixation device applied to rat femur.

Fracture slit adjusted to $2 \mathrm{~mm}$.

\section{Histomorphometric examination}

The specimens were stained with Alcian blue and hematoxylin/eosin, and then the area around the fracture gap was morphometrically examined in two central sections from each specimen using an image analysis system (Easy Image 2000 ; Bergström Instrument AB, Solna, Sweden). A standardised rectangular frame $(6 \times 5 \mathrm{~mm})$ was placed centrally over the fracture gap. Areas of new bone (woven and lamellar) and chondroid tissue were measured in the periosteal and intramedullary zones within the frame. Periosteal bone was defined as an area surrounded by membrane-like tissue located outside cortical bone. Medullary bone was defined as an area with osteogenic cells located inside cortical bone. Chondroid was defined as areas that stained positively with Alcian blue. Mean values were calculated for each area in two sections from each specimen, and are presented as $\mathrm{mm}^{2}$.

\section{Immunohistochemistry}

Two sections were stained in each specimen. Sections ( $5 \mu \mathrm{m}$ thick) were deparaffinised and antigens were exposed by microwave heating at $750 \mathrm{~W}$ for $190 \mathrm{~s}$ in $0.01 \mathrm{M}$ citric acid ( $\mathrm{pH} \mathrm{6.0)}$. The sections were cooled for $5 \mathrm{~min}$ in PBS, immersed in $0.3 \% \mathrm{H}_{2} \mathrm{O}_{2}$ in methanol for $1 \mathrm{~h}$, washed three times for $5 \mathrm{~min}$ in PBS and incubated with $3 \%$ rabbit serum in PBS-triton for $1 \mathrm{~h}$. The sections were then incubated for $12 \mathrm{~h}$ with monoclonal anti-rat ED2 (tissue macrophages) antibody (Serotec, Oxford, UK) in moisture chambers at $4^{\circ} \mathrm{C}$, washed twice in PBS for 5 min each and incubated in PBStriton for $1 \mathrm{~h}$ with a biotinylated rabbit anti-mouse
IgG antibody (DAKO, Copenhagen, Denmark) linked to peroxidase. The sections were washed in PBS three times for 5 min each, incubated in ABC solution for $1 \mathrm{~h}$, and then washed twice in PBS for 5 min each.

Peroxidase labelling was visualised by incubating the specimens in 3,3'diaminobenzidine (DAB ; $10 \mathrm{mg} / 15 \mathrm{~mL} \mathrm{PBS}$ ) for $10 \mathrm{~min}$ at room temperature followed by $0.02 \% \mathrm{H}_{2} \mathrm{O}_{2}$ in $\mathrm{PBS}$ for 5 min. The sections were rinsed in PBS for $5 \mathrm{~min}$ and finally dehydrated in an ethanol series and mounted. The numbers of cells that stained positively for the ED2 antibody were counted within a standardised rectangular frame $(0.8 \times 0.8 \mathrm{~mm})$ placed over the periosteum and the muscle tissue close to the fracture gap using an image analysis system (Easy Image 2000, Bergström Instrument AB, Solna, Sweden). Four areas of each section were counted. The mean and standard deviation were used.

Results are expressed as cells $/ \mathrm{mm}^{2}$.

\section{Statistics}

All specimens were assessed by an independent observer in a blinded manner. Data were statistically evaluated using the Mann-Whitney rank sum test.

We could not calculate significance levels between groups with similar results due to the low number of animals in each test group.

\section{RESULTS}

\section{Morphologic characteristics}

The morphology is described for all groups since microscopic evaluation did not identify any obvious differences in the morphological composition of the tissues among them.

Week 1 : (Control ; Fig. 2a). Mesenchymal cells and vessels formed dense tissues in the periosteal zone except in the centre where polymorphonuclear cells and lymphocytes continued to infiltrate necrotic tissue. Periosteal bone had formed de novo adjacent to the periosteum. Chondrocyte-like cells and blue-stained chondroid matrix were occasionally located adjacent to periosteal woven bone. Osteoclastic resorption and mesenchymal cells covered bone fragments in the intercortical zone and areas of new intramedullary bone were located in the marrow canal on both sides of the fracture.

Week 4: (Control ; Fig. 3). The centre of the fracture gap was dominated by chondroid cells and 


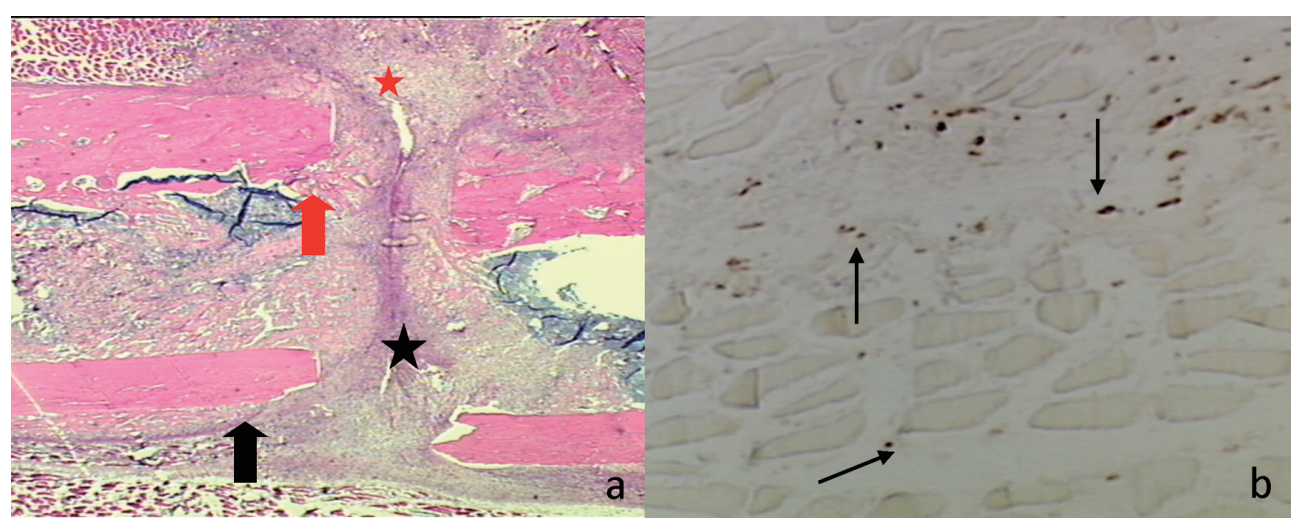

Fig. 2. Photographs of sections of control femur at one week after fracture.

a. Red star: Mesenchymal cells and vessels in periosteal region. Black star: Necrotic tissue and inflammatory cells. Black arrow: Periosteal new bone. Red arrow: Active bone resorption.

b. Arrows : Cells are stained positively for ED2 antibody.

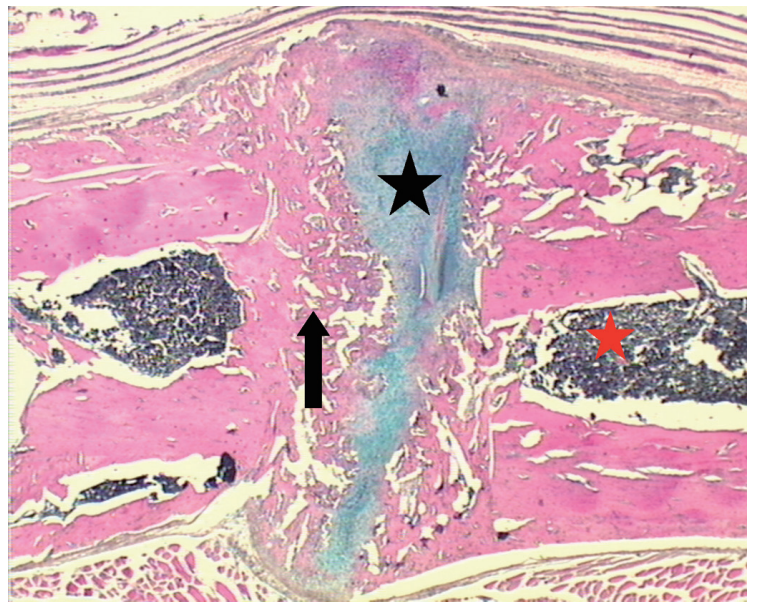

Fig. 3. Photographs of sections of femur of control at 4 weeks after fracture.

Star: Chondroid matrix contains chondroid cells. Black arrow : New intramedullary bone. Red star: Marrowlike tissue.

blue-stained chondroid matrix. Large areas of periosteal and intramedullary new bone with active osteoblasts were located adjacent to the chondroid tissue. Periosteal and/or intramedullary bone occasionally bridged the fracture gap. Marrow-like tissue was identified in the intramedullary zones but the marrow canal was not reconstituted.

\section{Histomorphometry}

Periosteal bone formation: After healing for 1 and 4 weeks, the area of periosteal de novo bone formation at the fracture site did not significantly differ between the low-dose and control groups. On the other hand, significantly less periosteal new bone was produced in the high-dose group after 1 week than in the control and low-dose groups $(p<0.01$; Fig. 4$)$.

Intramedullary bone formation: The areas of intramedullary bone did not significantly differ among the five groups at 1 week after surgery or sham treatment, or between the low-dose and control groups at 4 weeks (Fig. 5).

Chondroid tissue formation: Areas of chondroid tissue were negligible in a few specimens at 1 week, but similarly increased in the low-dose and control groups at week 4 (Fig. 6).

\section{Immunohistochemistry}

Cells that stained positively for ED2 antibodies were diffused throughout muscle tissue close to the fracture (Fig. 2b).

The control and high-dose groups contained the least and most (53 \pm 25 vs. $219 \pm 49)$ ED2-stained cells $/ \mathrm{mm}^{2}$, respectively. The low-dose group contained $129 \pm 25$, ED2-positive cells $/ \mathrm{mm}^{2}$. The numbers of macrophages $/ \mathrm{mm}^{2}$ significantly differed among all three groups $(p<0.001$; Fig. 7$)$.

\section{DISCUSSION}

The present results indicated that fracture healing in healthy rats, including de novo periosteal and medullary bone and chondroid tissue, is unaffected by ongoing therapy with low-dose MTX, whereas high-dose MTX negatively influences bone formation, resulting in significantly less areas of new periosteal bone. The impact of MTX was obvious in terms of the density of ED2-positive cells adjacent to the fracture gap; the numbers of these cells were increased in both MTX groups compared with the control group.

The process of fracture healing generally res- 


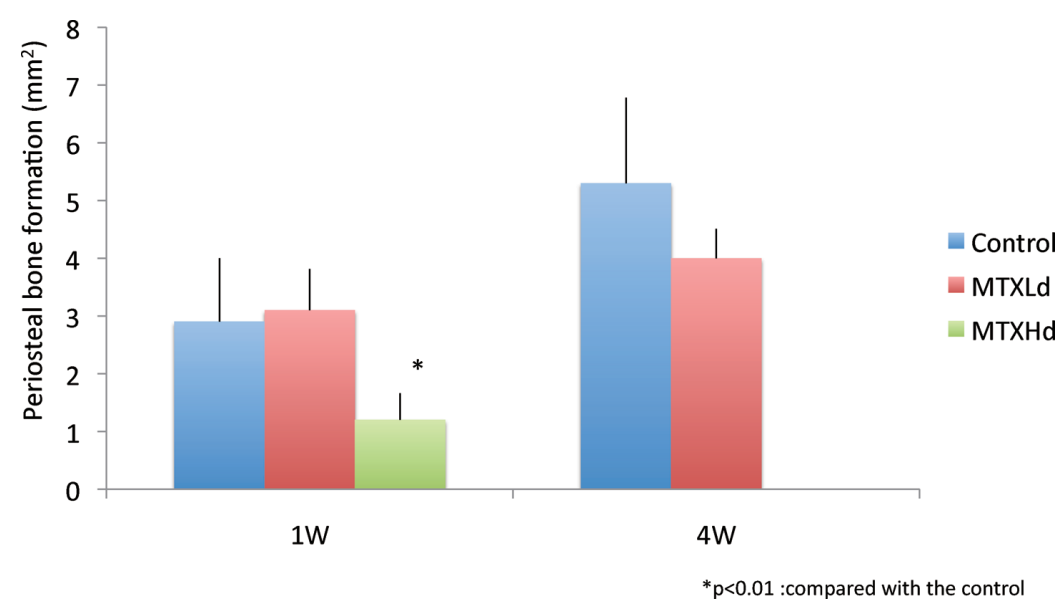

Fig. 4. Formation of new periosteal bone at 1 and 4 weeks after fracture.

*Significantly less new bone in HMD than in control (Con1) group $(p<0.01)$

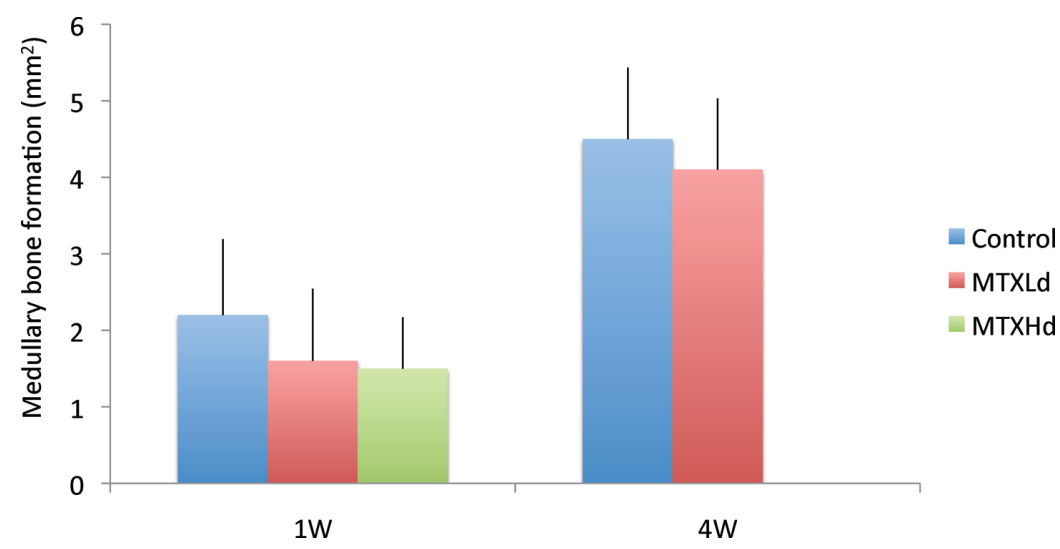

Fig. 5. Formation of medullary bone at 1 and 4 weeks after fracture. Bone production does not significantly differ among groups.

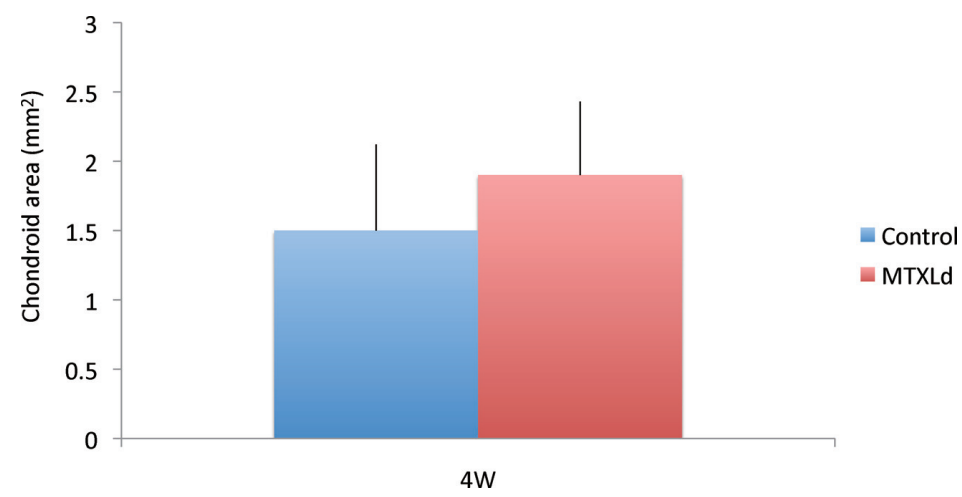

Fig. 6. Formation of chondroid matrix at 4 weeks after fracture. Control (Con4) and LDM4 groups do not significantly differ.

tores the structure and function of traumatised bone. A fracture hematoma that initially forms after fracture of a long bone is followed by inflammation and neovascularisation. Undifferentiated mesenchymal cells migrate into the damaged area during endochondral bone formation and differentiate into chondrocytes to produce a cartilage matrix. Vascular buds infiltrate the matrix and osteoprogenitor cells are thought to accompany these neovessels. Finally, the osteoprogenitor cells differentiate into 


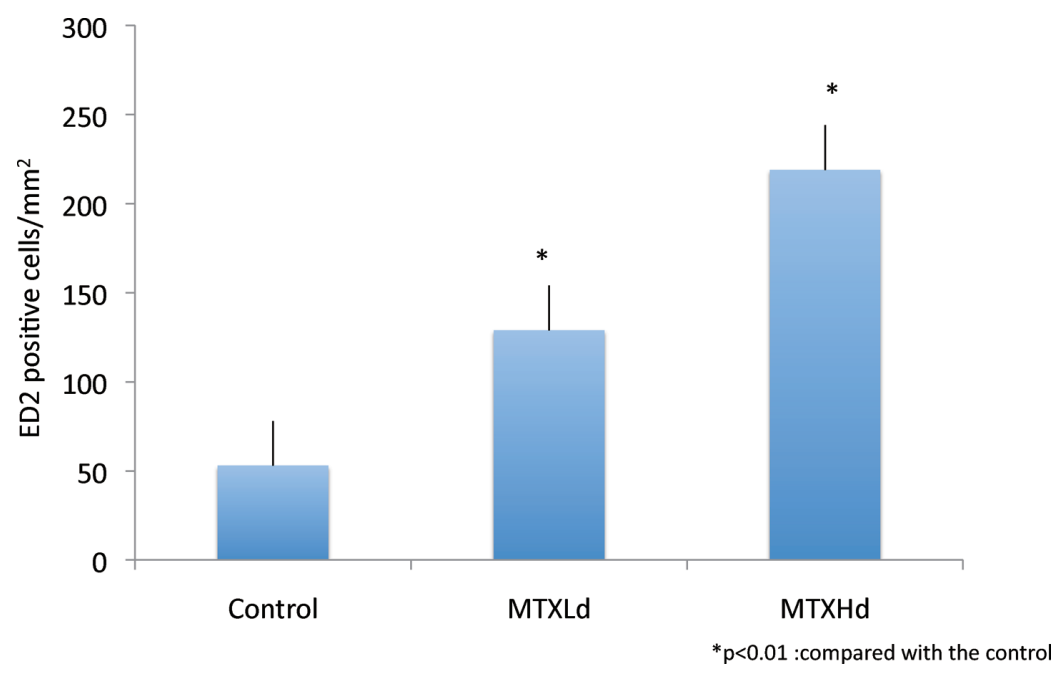

Fig. 7. Total number of cells $/ \mathrm{mm}^{2}$ positively stained for ED2 (surface antigen on tissue macrophages) antibody at one week after fracture.

*Both MTX groups significantly differ from control.

osteoblasts and form bone matrix.

Macrophages and polymorphonuclear leucocytes migrate into fractured areas from adjacent tissues during the early inflammatory phase of fracture healing. Activated macrophages when present in a fracture hematoma participate in the debridement of necrotic tissues and also in regulating the concentration of proinflammatory cytokines such as IL 1, IL 6 and TNFa. However, the role of macrophages seems to be more complex than simply that of a participant in the inflammatory process. The activation of macrophages in healing fractures results in an immature callus with low biomechanical rigidity ${ }^{12)}$. These results emphasise that macrophages not only participate in necrotic tissue debridement, but also interfere with osteoprogenitor cell differentiation.

Little is understood about the effects of lowdose MTX on the healing of fractured bone, whereas high doses clearly induce deleterious effects. Healed femoral fractures in rats given high doses of MTX are not as strong as those of control rats ${ }^{13)}$.

The effects of low-dose MTX on bone metabolism when administered as a DMARD are more complex and difficult to evaluate, since systemic inflammatory diseases such as RA negatively affect not only the joints, but also the processes of bone turnover and remodelling. Thus, osteoporosis and periarticular decalcification are established features of rheumatoid arthritis ${ }^{14)}$. Inflammatory cytokines most probably mediate these processes, but physical inactivity and treatment with corticosteroids might also be part of the explanation.

The effects of low-dose MTX on bone metabo- lism in experimental animals and models in vitro have been investigated. May demonstrated the development of osteopenia, osteoclast recruitment and the suppression of osteoblast activity in rats after 16 weeks of low-dose MTX ${ }^{15,16}$. Laurindo et al. demonstrated a negative effect of MTX on the bone mineral density of healthy, growing rabbits ${ }^{17}$.

However, low-dose MTX might positively affect bone turnover in an animal model of adjuvant arthritis since it restores the osteogenic activity of bone marrow cells reduced by arthritis ${ }^{9}$. Furthermore, MTX does not affect the proliferation and differentiation of osteoblasts ${ }^{18}$. One possible interpretation of these differing experimental results is that lowdose MTX exerts a negative effect on bone metabolism in otherwise healthy individuals, whereas it might have a positive influence in patients with inflammatory arthritis, possibly through counteracting its catabolic effects. Even though high-dose MTX was injected as a bolus, the slower progress of new periosteal bone formation in the high-dose group in the present study indicated that MTX negatively influences bone regeneration at higher doses. These results are consistent with the findings published by Pelker et al.

However, our results in rats administered with low-dose MTX seem contradictory to findings in rats and rabbits in vivo ${ }^{16,17)}$. The present study did not find any significantly negative effects of a low dose of MTX on endochondral fracture healing in healthy rats at both 1 and 4 weeks of followup. The explanation for these differing results might lie in the regulatory mechanisms of normal bone turnover versus actual fracture healing. Bone 
healing is precisely controlled, given that environmental factors remain constant throughout the process $^{18)}$. The findings of a study of de novo vessels invading cartilage matrix in fractures indicate that endochondral ossification recapitulates the processes that occur in the growth plates of long bones during embryonic development ${ }^{19)}$. Accordingly, these processes are probably regulated quite differently and are thus differentially susceptible to environmental strain. Areas of bone formation were evaluated in the present study, but bone density and strength were not compared after MTX administration. Further studies are required to determine the strength of new bone formed in the presence and absence of MTX.

One unusual finding in the present study was the increased numbers of ED2-positive cells in both MTX groups. However, we could not evaluate the mechanism or the extent to which the increased concentration of monocytes/macrophages affects the repair process. The whole area of inflammation is also important. However, because the inflammatory border could not be defined, the numbers of macrophages in such areas were counted. It is reported that macrophages increase after fracture, and decrease from the fracture area at the beginning of the bone formation in the rat experimental fracture healing study ${ }^{20)}$. Our results indicated that a moderate increase in the number of macrophages in a fracture area does not negatively influence the repair process expressed as new bone formation. However, a more pronounced increase, such as that in the high-dose group, might negatively influence the formation of new periosteal bone. The etiology behind the increased macrophage concentration is not easily explained. It might be due to MTX effects on the production of local factors (for example chemokines) that attract monocytes to areas of inflammation. The anti-inflammatory effect of MTX might also prolong the initial inflammatory phase of fracture healing. Thus, MTX might not increase the number of attracted monocytes/macrophages but rather prolong their presence in the fracture area. Osteoclasts are differentiated by monocytes and break up into monocyts after completing their resorptive activity ${ }^{21)}$. According to the osteroclats activity, transient expression of macrophage might be pronounced. In addition, the number of macrophages examined only one time point in this study, therefore, the chronological changes of macrophages expression has not investigated. This is the limitation of this study. Either explanation might be valid, but the eventual effects of this phe- nomenon on the healing process and the quality of new bone could not be evaluated within the framework of this study.

The present study indicates that a low dose of MTX does not affect endochondral fracture healing in healthy rats, whereas a high dose seems to negatively affect the formation of new periosteal bone. Methotrexate induced a periosteal increase in the numbers of ED2-positive cells. The impact of this increase on inflammatory reactions in the fracture area and on the process of bone repair remains unknown. Further investigation is required to elucidate the mechanisms behind this phenomenon.

\section{ACKNOWLEDGEMENTS}

This study was supported by grants from the Gothenburg Medical Society, the Gothenburg Rheumatology association and the Swedish Rheumatology association.

\section{REFERENCES}

1. Chan ES, Cronstein BN. Molecular action of methotrexate in inflammatory diseases. Arthritis Res, 4 : 266-273, 2002.

2. Nesbit M, Krivit W, Heyn R, Sharp H. Acute and chronic effects of methotrexate on hepatic, pulmonary and skeletal systems. Cancer, 37: 10481054, 1976.

3. Ragab AH, Frech RS, Vietti TJ. Osteoporotic fractures secondary to methotrexate therapy of acute leukaemia in remission. Cancer, 25 : 580$585,1970$.

4. Bridges SL Jr, Moreland LW. Perioperative use of methotrexate in patients with rheumatoid arthritis undergoing orthopedic surgery. Rheum Dis Clin North Am, 23 : 981-993, 1997.

5. Carpenter MT, West SG, Vogelgesang SA, Casey Jones DE. Postoperative joint infections in rheumatoid arthritis patients on methotrexate therapy. Orthopedics, 19 : 207-210, 1996.

6. Escalante A, Beardmore TD. Risk factors for early wound complications after orthopedic surgery for rheumatoid arthritis. J Rheumatol, 22 : 1844-1851, 1995.

7. Mark H, Bergholm J, Nilsson A, Rydevik B, Stromberg L. An external fixation method and device to study fracture healing in rats. Acta Orthop Scand, 74 : 476-482, 2003.

8. Mark H, Nilsson A, Nannmark U, Rydevik B, Bergholm J, Stromberg L. Effects of fracture fixation stability on ossification in healing fractures. An external fixation method and device to 
study fracture healing in rats. Clin Orthop, 74 : 245-250, 2004.

9. Suzuki Y, Nakagawa M, Masuda C, Ide M, Uehara R, Ichikawa Y, Mizushima Y. Short-term low dose methotrexate ameliorates abnormal bone metabolism and bone loss in adjuvant induced arthritis. J Rheumatol, 24 : 1890-1895, 1997.

10. Scheufler E. Evidence for Nonlinear Pharmacokinetics of Methotrexate in the Rat. Pharmacology, 25 : 51-56, 1982.

11. Sarah L. Morgan, et al. MTX Affects Inflammation and Tissue Destruction Differently in the Rat AA Model. J Rheuma, 28 : 1476-1481, 2001.

12. Grundnes O, Reikeraas O. Effects of macrophage activation on bone healing. J Orthop Sci, 5 : 243247, 2000.

13. Pelker RR, Friedlaender GE, Panjabi MM, Markham T, Hausman M, Doganis AC, McKay J. Chemotherapy-induced alterations in the biomechanics of rat bone. J Orthop Res, 3: 91-95, 1985.

14. Reid DM, Harvie J, England AJ, Garton MJ, Nicoll JJ, Tothill P, Nuki G, Smith MA, Higgins B, Kennedy NS, Nicoll J, Brown N. Corticosteroidinduced osteoporosis: guidelines for prevention — are they useful? Br J Rheumatol, 3 : 10351037, 1997.
15. May KP, Mercill D, McDermott MT, West SG. The effect of methotrexate on mouse bone cells in culture. Arthritis Rheum, 39 : 489-494, 1996.

16. May KP, West SG, McDermott MT, Huffer WE. The effect of low-dose methotrexate on bone metabolism and histomorphometry in rats. Arthritis Rheum, 37 : 201-206, 1994.

17. Laurindo I, Mendes F, Novaes G, Mello S, Falco V, Jorgetti V. Methotrexate inhibition of bone mineral density increase in growing rabbits: prevention by folinic acid. Clinical and Experimental Rheumatology, 21 : 581-586, 2003.

18. Minaur NJ, Jefferiss C, Bhalla AK, Beresford JN. Methotrexate in the treatment of rheumatoid arthritis. I. In vitro effects on cells of the osteoblast lineage. Rheumatology, 41 : 735-740, 2002.

19. Mark H, Messina A, Nannmark U, Morrison W, Penington A. Microvascular invasion during endochondral ossification in experimental fractures in rats. Bone, 35 : 535-42, 2004.

20. Henricson A, Hukth A, Johnell O. The occurrence of accessory immunologic cells in bone induction. Clin Orthop Rel Res, 264 : 270-277, 1991.

21. Buckwalter JA, Glimcher MJ, Cooper RR, Recker R. Bone Biology. JBJS (Am), 77 : 1256-1275, 1995. 